\title{
Aplicação de análise estatística para identificação de tendências climáticas(1)
}

\begin{abstract}
Álvaro José Back(2)
Resumo - O objetivo deste trabalho foi usar a análise estatística para identificar tendências anuais da temperatura e precipitação pluvial. Foi usada a série histórica de 1924 a 1998 de precipitação pluvial e temperatura média do ar à superfície, da estação meteorológica de Urussanga (latitude 2831' S, longitude 49 $19^{\prime} \mathrm{W}$ e altitude de 48,2 m). Foram empregados a análise de regressão e os testes paramétricos de Run, Mann-Kendall e Pettitt. Os resultados indicam que houve tendência significativa no aumento da temperatura média anual e na temperatura média do mês de janeiro, sendo que a mudança ocorreu no ano de 1965. Não foi identificada nenhuma tendência significativa na temperatura média do mês de julho. Também foi identificada a tendência significativa de aumento da precipitação pluvial total anual, e da precipitação pluvial total no quarto trimestre. Nos três primeiros trimestres do ano, nenhuma tendência significativa foi identificada.
\end{abstract}

Termos para indexação: mudança climática, precipitação, água de chuva, temperatura do ar.

\section{Application of statistical analysis for identification of climatic tendency}

\begin{abstract}
With the objective to give a statistical treatment in the identification of climatic change and annual tendency, time serie from 1924 to 1998 of rainfall and surface air temperatures for the meteorological station of Urussanga (latitude $28^{\circ} 31^{\prime} \mathrm{S}$, longitude $49^{\circ} 19^{\prime} \mathrm{W}$ and altitude of $48.2 \mathrm{~m}$ ) was analyzed. The analyses were made through regression analyses and the non-parametric tests of Run, Mann-Kendall and Pettitt. The results indicate warming trends for the mean annual temperature and for January mean temperature, with the change point in 1965 . July mean temperature did not present any significant trend. The total annual rainfall and the total rainfall in the fourth trimester of the year presented significant tendency of increasing. For the first three trimesters no significant trend was detected on the total rainfall.
\end{abstract}

Index terms: climatic change, precipitation, rainwater, air temperature.

\section{Introdução}

Uma das grandes preocupações da comunidade científica na última década diz respeito às alterações climáticas e suas conseqüências para a humanidade. Foi constatado que a temperatura média global aumentou entre 0,3 e $0,6^{\circ} \mathrm{C}$ desde o final do século passado (Houghton et al., 1996). Nos últimos anos, tem sido muito discutida a possibilidade de mudança climática como conseqüência da emissão de gases de efeito-estufa pelas atividades humanas (Bruce, 1990; Berlato et al., 1995).

\footnotetext{
(1) Aceito para publicação em 15 de agosto de 2000 .

(2) Empresa de Pesquisa Agropecuária e Extensão Rural de Santa Catarina (EPAGRI), Estação Experimental de Urussanga, Caixa Postal 49, CEP 88840-000 Urussunga, SC. E-mail: ajb@epagri.rct-sc.br
}

As conseqüências do efeito-estufa no aquecimento global foram analisadas e discutidas por Houghton et al. (1990, 1992) e Houghton et al. (1996) e National Research Council (1992). Esses trabalhos concluíram que as mudanças observadas no clima global não são ainda suficientemente grandes para serem atribuídas inequivocamente a causas antropogênicas de aumento do efeito-estufa. Karl et al. (1996), estudando dados climáticos dos Estados Unidos, concluíram que, embora a temperatura tenha apresentado substancial incremento, as alterações não são grandes, nem temporalmente consistentes para se rejeitar a hipótese de que as mudanças tenham ocorrido por variações aleatórias naturais.

$\mathrm{O}$ aquecimento global tem como conseqüências diretas a alteração na freqüência e distribuição das chuvas, aumentando as ocorrências de secas e de cheias (Karl et al., 1996). Dai et al. (1997) apresentaram estudos sobre a variação da distribuição da preci- 
pitação na superfície do planeta no período de 1900 a 1988, mostrando que em grande parte houve um aumento da precipitação, porém em outras regiões foi registrada uma diminuição da precipitação.

Várias projeções, com diferentes cenários e taxas de emissão de gases pela atividade humana que causam o efeito-estufa, têm previsto um aquecimento global na superfície terrestre. Outras alterações previstas nessas simulações são o aumento da precipitação, maior ocorrência de precipitações intensas originadas por processos convectivos, maior freqüência de cheias e ocorrências de secas mais severas e mais prolongadas (Houghton et al., 1996; Karl et al., 1996).

Por outro lado, muitas tendências de aquecimento observadas nos registros meteorológicos expressam mais os efeitos urbanos locais do que o aquecimento global da atmosfera. O surgimento das chamadas "ilhas de calor" devido à urbanização produz o aquecimento da atmosfera que afeta os registros de temperatura, uma vez que a maioria das estações meteorológicas se encontram próximas ao centros urbanos. Existem vários trabalhos mostrando que o impacto da urbanização pode superar o aquecimento global (Jones et al., 1986; Hansen \& Lebedeff, 1987; Karl et al., 1988).

A identificação de alterações nos registros meteorológicos é de grande importância para os estudos de engenharia que utilizam as séries históricas, pois tanto as simulações como as aplicações de teorias de probabilidade são realizadas com a hipótese de que as séries históricas são homogêneas, isto é, que não apresentem tendências. Uma série é dita homogênea quando os dados vêm da mesma população, e assim não há alteração nos parâmetros.

Yevjevich (1972) define tendência em uma série temporal como uma mudança sistemática e contínua em qualquer parâmetro de uma dada amostra, excluindo-se mudanças periódicas ou quase periódicas Goossens \& Berger (1986) apresentam as definições de alguns termos usados para caracterizar modificações climáticas. Segundo esses autores, mudança climática é um termo completamente geral que engloba todas as formas de inconstâncias climáticas de natureza estatística ou de causas físicas. Flutuação climática é qualquer forma de mudança sistemática regular ou irregular, exceto a tendência e mudança abrupta. Oscilação climática é a flutuação na qual a variável tende a mover-se gradualmente e de forma suave entre sucessivos máximos e mínimos. A tendência climática é uma mudança climática caracterizada por um suave acréscimo ou decréscimo nos valores médios no período de registro. A mudança climática abrupta é uma mudança abrupta e permanente, durante o período de registro, de um valor médio para outro.

O problema da existência ou não de tendência nas séries de temperatura e precipitação pluvial no Estado de Santa Catarina tem sido objeto de muitas especulações, principalmente devido às enchentes que ocorreram nas décadas de 80 e 90 . Não existem estudos suficientes para comprovar estatisticamente a existência de tendências nas séries climatológicas nesse Estado. A dificuldade no estabelecimento de existência de tendência está na grande variabilidade natural dos dados meteorológicos.

Este trabalho visa usar a análise estatística para identificar tendências anuais da temperatura e precipitação pluvial.

\section{Material e Métodos}

Foram utilizados dados de precipitação mensal e temperatura média mensal da estação meteorológica de Urussanga, SC, relativos ao período de 1924 a 1998. A estação meteorológica, classificada como Estação Climatológica Principal, pertence à rede meteorológica da Empresa Catarinense de Pesquisa Agropecuária (Epagri), e está localizada na estação experimental (latitude $28^{\circ} 31^{\prime} \mathrm{S}$, longitude $49^{\circ} 19^{\prime} \mathrm{W}$, com altitude de $48,2 \mathrm{~m}$ ), próximo ao centro urbano de Urussanga com aproximadamente 10.000 habitantes. No período anterior a 1955, a estação estava em local distante aproximadamente $2 \mathrm{~km}$ do atual.

$\mathrm{Na}$ avaliação da precipitação foram considerados os totais anuais e totais por trimestre do ano, e em relação à temperatura média foram consideradas a temperatura média anual e a temperatura média dos meses de janeiro e julho. Em cada variável estudada, foram aplicadas a análise de regressão, e realizadas análises não-paramétricas por meio dos testes Run, de Mann-Kendall e de Pettitt.

$O$ teste Run é um teste não-paramétrico apresentado por Thom (1966), que é usado para avaliar se uma série ocorre aleatoriamente. Consiste em realizar a contagem do número de oscilações dos valores acima e abaixo da mediana, numa série de dados naturalmente ordenada. O número de oscilações é chamado de Run, e deve-se testar se o valor 
observado está dentro da faixa de distribuição considerada normal. Um valor alto de Run indica muitas oscilações, e valores baixos indicam um desvio em relação à mediana durante o período de registros.

Se a seqüência contém $\mathrm{N}_{1}$ símbolos de um tipo, e $\mathrm{N}_{2}$ símbolos de outro tipo (e $\mathrm{N}_{1}$ e $\mathrm{N}_{2}$ não são muito pequenos), a distribuição amostral do número de Runs total pode ser aproximada pela distribuição normal com média:

$$
\mathrm{E}(\mathrm{u})=\frac{2 \mathrm{~N}_{1} \mathrm{~N}_{2}}{\mathrm{~N}_{1}+\mathrm{N}_{2}}+1
$$

e a variância da distribuição pode ser estimada por

$\operatorname{Var}(\mathrm{u})=\frac{2 \mathrm{~N}_{1} \mathrm{~N}_{2}\left(2 \mathrm{~N}_{1} \mathrm{~N}_{2}-\mathrm{N}_{1}-\mathrm{N}_{2}\right)}{\left(\mathrm{N}_{1}+\mathrm{N}_{2}\right)^{2}\left(\mathrm{~N}_{1}+\mathrm{N}_{2}-1\right)}$

onde u representa o número de Runs. Por isso, a hipótese nula de que a distribuição dos símbolos ocorre normalmente, e que a amostra é aleatória, pode ser testada com base na estatística:

$\mathrm{z}=\frac{\mathrm{u}-\mathrm{E}(\mathrm{u})}{\sqrt{\operatorname{var}(\mathrm{u})}}$

Este valor calculado pode ser comparado com valores de z para distribuição normal. Para o nível de significância de $5 \%$, z deve estar entre $-1,96$ e 1,96. Caso z calculado seja maior que o valor tabelado, deve-se rejeitar a hipótese de nulidade.

A análise de regressão pode ser utilizada para indicar alterações climáticas por meio do teste de significância do coeficiente angular. Considerando a equação da reta do tipo:

$\mathrm{Y}=\mathrm{aX}+\mathrm{b}$,

o teste consiste em determinar o intervalo de confiança do coeficiente a, sendo que se este intervalo não inclui o valor zero, a tendência é significativa.

O teste de Mann-Kendall, proposto inicialmente por Sneyers (1975), considera que, na hipótese de estabilidade de uma série temporal, a sucessão de valores ocorre de forma independente, e a distribuição de probabilidade deve permanecer sempre a mesma (série aleatória simples). Goossens \& Berger (1986) afirmam que o teste de MannKendall é o método mais apropriado para analisar mudanças climáticas em séries climatológicas e permite também a detecção e localização aproximada do ponto inicial de determinada tendência.

Moraes et al. (1995) descrevem o método considerando uma série temporal de $\mathrm{Xi}$ de $\mathrm{N}$ termos $(1 \leq \mathrm{i} \leq \mathrm{N})$; o teste consiste na soma $t_{n}$ do número de termos $m_{i}$ da série, relativo ao valor $\mathrm{X}_{\mathrm{i}}$ cujos termos precedentes $(\mathrm{j}<\mathrm{i})$ são inferi- ores ao mesmo $\left(\mathrm{X}_{\mathrm{j}}<\mathrm{X}_{\mathrm{i}}\right)$, isto é:

$$
\mathrm{t}_{\mathrm{n}}=\sum_{\mathrm{i}=1}^{\mathrm{n}} \mathrm{m}_{\mathrm{i}} \text {. }
$$

Para séries com grande número de termos $(\mathrm{N})$, sob a hipótese nula (Ho) de ausência de tendência, $\mathrm{t}_{\mathrm{n}}$ apresentará uma distribuição normal com média e variância:

$$
\begin{aligned}
& E\left(t_{n}\right)=\frac{N(N-1)}{4} \\
& \operatorname{Var}\left(t_{n}\right)=\frac{N(N-1)(2 N+5)}{72} .
\end{aligned}
$$

Testando a significância estatística de $t_{n}$ para a hipótese nula usando um teste bilateral, esta pode ser rejeitada para grandes valores da estatística $\mathrm{u}(\mathrm{t})$ dada por:

$\mathrm{u}(\mathrm{t})=\frac{\left(\mathrm{t}_{\mathrm{n}}-\mathrm{E}\left(\mathrm{t}_{\mathrm{n}}\right)\right)}{\sqrt{\operatorname{Var}\left(\mathrm{t}_{\mathrm{n}}\right)}}$.

O valor da probabilidade $\alpha_{1}$ é calculado por meio de uma Tabela da normal reduzida tal que:

$\alpha_{1}=\operatorname{prob}(|\mathrm{u}|>|\mathrm{u}(\mathrm{t})|$

a hipótese nula é rejeitada, ou não, a um nível de significância $\alpha_{0}$ se $\alpha_{1}>\alpha_{0}$ ou $\alpha_{1}<\alpha_{0}$, respectivamente. Em geral, considera-se o nível de significância do teste $\alpha_{0}=0,005$. A hipótese nula é rejeitada quando existe uma tendência significativa na série temporal. $\mathrm{O}$ sinal da estatística $\mathrm{u}(\mathrm{t})$ indica se a tendência é crescente $(\mathrm{u}(\mathrm{t})>0)$ ou decrescente $(\mathrm{u}(\mathrm{t})<0)$

O ponto de início da mudança pode ser determinado aplicando-se o mesmo princípio à série inversa. Neste caso, computa-se para cada termo o número de termos $\mathrm{m}{ }_{\mathrm{i}}$ da série $\mathrm{X}_{\mathrm{j}}$, de maneira tal que para $\mathrm{X}_{\mathrm{i}}>\mathrm{X}_{\mathrm{j}}$ com $\mathrm{i}<\mathrm{j}$ os valores de $u^{\prime}(\mathrm{t})$ para a série retrógrada sejam dados por:

$u^{*}\left(d_{i}\right)=-u\left(d_{i}\right)$

A interseção das curvas $u(t)$ e $u^{*}(t)$ localiza o ponto de mudança, se esta ocorre dentro dos valores críticos do intervalo de confiança.

O teste de Pettitt (Pettitt, 1979; Moraes et al., 1995) utiliza uma versão do teste de Mann-Whitney, no qual se verifica se duas amostras $\mathrm{X}_{1}, \ldots . \mathrm{X}_{\mathrm{t}}$ e $\mathrm{X}_{\mathrm{t}+1}, \ldots ., \mathrm{X}_{\mathrm{T}}$ são da mesma população. A estatística $U_{t, T}$ faz uma contagem do número de vezes que um membro da primeira amostra é maior que o membro da segunda, e pode ser escrita:

$\mathrm{U}_{\mathrm{t}, \mathrm{T}}=\mathrm{U}_{\mathrm{t}-1, \mathrm{~T}}+\sum_{\mathrm{j}=1}^{\mathrm{T}} \operatorname{sgn}\left(\mathrm{X}_{\mathrm{i}}-\mathrm{X}_{\mathrm{j}}\right) \quad$ para $\mathrm{t}=2, . ., \mathrm{T}$ 
onde: $\operatorname{sgn}(x)=1$ para $x>0 ; \operatorname{sgn}(x)=0$ para $x=0 ; \operatorname{sgn}(x)=-1$ para $\mathrm{x}<0$.

A estatística $U_{t, T}$ é então calculada para os valores de $1<\mathrm{t}<\mathrm{T}$, e a estatística $\mathrm{k}(\mathrm{t})$ do teste de Pettitt se escreve:

$\mathrm{k}(\mathrm{t})=\mathrm{MAX}_{1 \leq \mathrm{t} \leq \mathrm{T}}\left|\mathrm{U}_{\mathrm{t}, \mathrm{T}}\right|$.

Esta estatística localiza o ponto onde houve uma mudança brusca na média de uma série temporal, e a sua significância pode ser calculada aproximadamente pela equação:

$\mathrm{p} \cong 2 \exp \left\{-6 \mathrm{k}(\mathrm{t})^{2} /\left(\mathrm{T}^{3}+\mathrm{T}^{2}\right)\right\}$

O ponto de mudança brusca é o t onde ocorre o máximo de $\mathrm{k}(\mathrm{t})$

Os valores críticos de $\mathrm{K}$ podem ser calculados pela equação:

Kcrit $= \pm \sqrt{\frac{-\ln (\mathrm{p} / 2)\left(\mathrm{T}^{3}+\mathrm{T}^{2}\right)}{6}}$.

\section{Resultados e Discussão}

Observa-se que pelo teste Run somente as séries de precipitação pluvial total anual e da temperatura média anual não podem ser consideradas como sendo homogêneas (Tabela 1). Pela análise de regressão, observa-se que há tendência significativa no aumento da precipitação total anual, da ordem de $3,4 \mathrm{~mm}$ por ano. Porém, quando analisada por trimestre, verifica-se que a tendência só é significativa no quarto trimestre do ano. Prudêncio et al. (1999), analisando dados de 32 estações pluviométricas do litoral de Santa Catarina, com séries de dados variando entre 1961 a 1995, constataram que em doze estações houve aumento significativo na precipitação total anual, em uma houve diminuição, e nas restantes não houve tendência significativa. Esses dados mostram que muitas alterações têm caráter regional, e não estão relacionadas a uma mudança global do clima. Berlato et al. (1995), estudando dados de precipitação de 17 locais do Rio Grande do Sul, não identificaram tendências de longo prazo na precipitação pluvial anual, ressaltando que as reduções e incrementos que se observaram em curto período se referem, possivelmente, à flutuação natural da precipitação pluvial.

A análise de regressão mostrou tendência significativa de aumento para a temperatura média anual (aproximadamente $1^{\circ} \mathrm{C} /$ século) e também para a temperatura média do mês de janeiro (aproximadamente $1,8^{\circ} \mathrm{C} /$ século). Esses valores são consistentes, embora superiores aos encontrados por Mota et al. (1993), que quantificou um incremento equivalente a $0,65^{\circ} \mathrm{C} /$ século para o Rio Grande do Sul. Também são superiores ao aquecimento global de $0,5^{\circ} \mathrm{C}$ no último século (Houghton et al., 1996). No entanto, Mello \& Alfonsi (1993) e Sentelhas et al. (1994) encontraram valores de aquecimento de $2^{\circ} \mathrm{C}$ em 90 anos na cidade de Campinas, SP. Esses maiores valores possivelmente refletem também o efeito da urbanização.

As formas gráficas do teste de Mann-Kendall e de Pettitt são apresentadas nas Figuras 1 a 8, onde as linhas horizontais representam os limites críticos do intervalo de confiança bilateral de $95 \%$. No teste de Mann-Kendall, a tendência é significativa quando os valores absolutos de $u(t)$ são maiores que os

Tabela 1. Resultados dos testes de Run e da análise de regressão linear.

\begin{tabular}{|c|c|c|c|}
\hline \multirow[t]{2}{*}{ Variável analisada } & \multirow{2}{*}{$\begin{array}{l}\text { Teste de Run } \\
\text { ( } \mathrm{Z} \text { calculado) }\end{array}$} & \multicolumn{2}{|c|}{ Análise de regressão } \\
\hline & & Coeficiente angular & Intervalo de confiança $(95 \%)$ \\
\hline Precipitação anual & $-2,575 *$ & 3,428 & 0,361 a $6,496^{*}$ \\
\hline Precipitação total no $1^{\circ}$ trimestre & $-0,234$ & 1,059 & $-0,668$ a 2,787 \\
\hline Precipitação total no $2^{\underline{0}}$ trimestre & 0,000 & $-0,202$ & $-1,366$ a 0,961 \\
\hline Precipitação total no $3^{-}$trimestre & $-0,234$ & 0,761 & $-0,697$ a 2,210 \\
\hline Precipitação total no $4^{0}$ trimestre & $-0,702$ & 1,767 & 0,281 a $3,254^{*}$ \\
\hline Temperatura média anual & $-3,043^{*}$ & 0,0098 & 0,0031 a $0,0165^{*}$ \\
\hline Temperatura média de janeiro & $-0,936$ & 0,0176 & 0,010 a $0,026^{*}$ \\
\hline Temperatura média de julho & $-0,722$ & 0,0013 & $-0,017$ a 0,019 \\
\hline
\end{tabular}

*Significativo a $5 \%$ de probabilidade. 

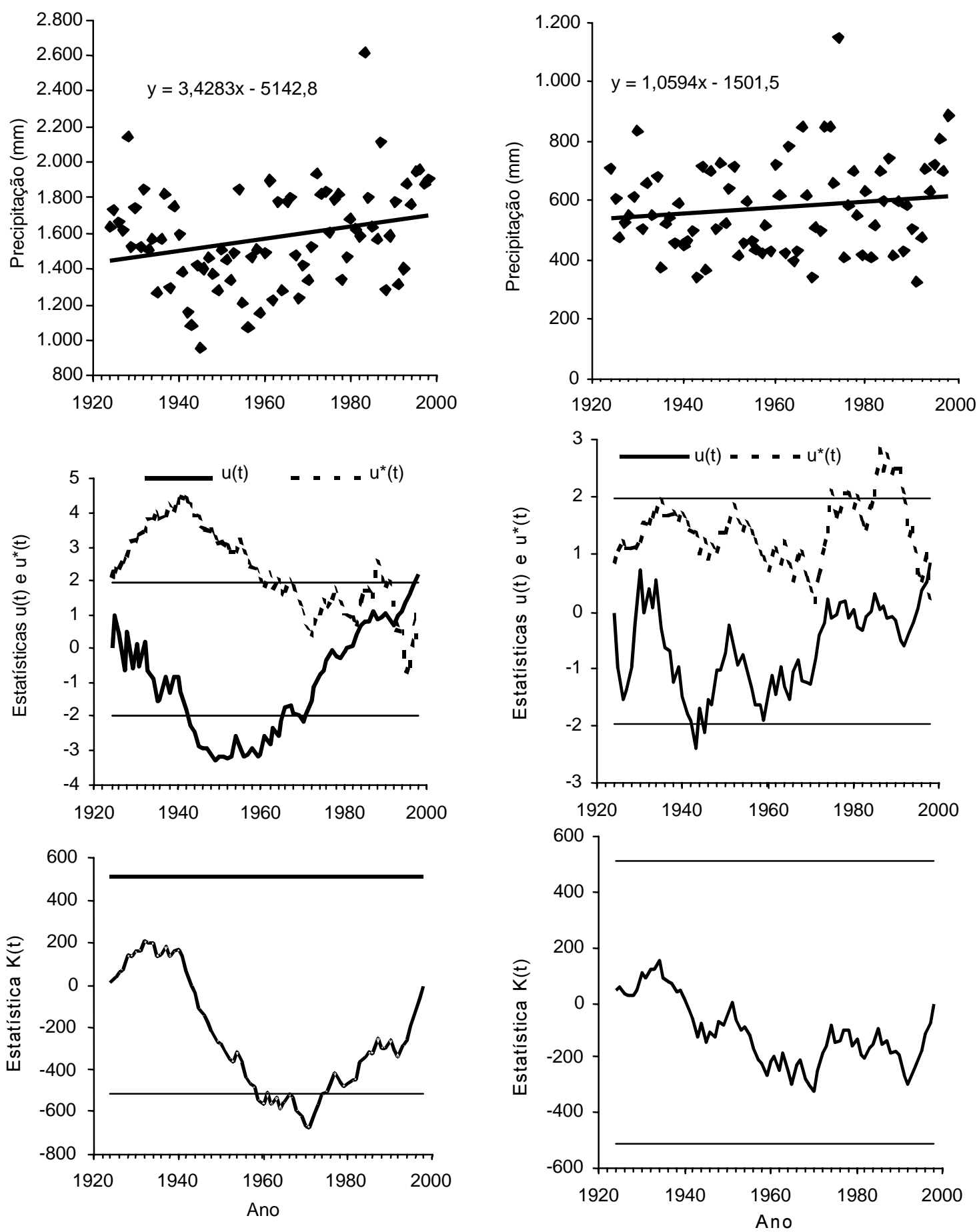

Figura 1. Precipitação total anual (regressão linear), estatísticas $\mathrm{u}(\mathrm{t})$ e $\mathrm{u}^{*}(\mathrm{t})$ do teste de Mann-Kendall e estatística $\mathrm{K}(\mathrm{t})$ do teste de Pettitt. As linhas horizontais representam o intervalo de confiança de $95 \%$.

Figura 2. Precipitação total no primeiro trimestre (regressão linear), estatísticas $u(t)$ e $u^{*}(t)$ do teste de MannKendall e estatística K(t) do teste de Pettitt. As linhas horizontais representam o intervalo de confiança de $\mathbf{9 5 \%}$. 

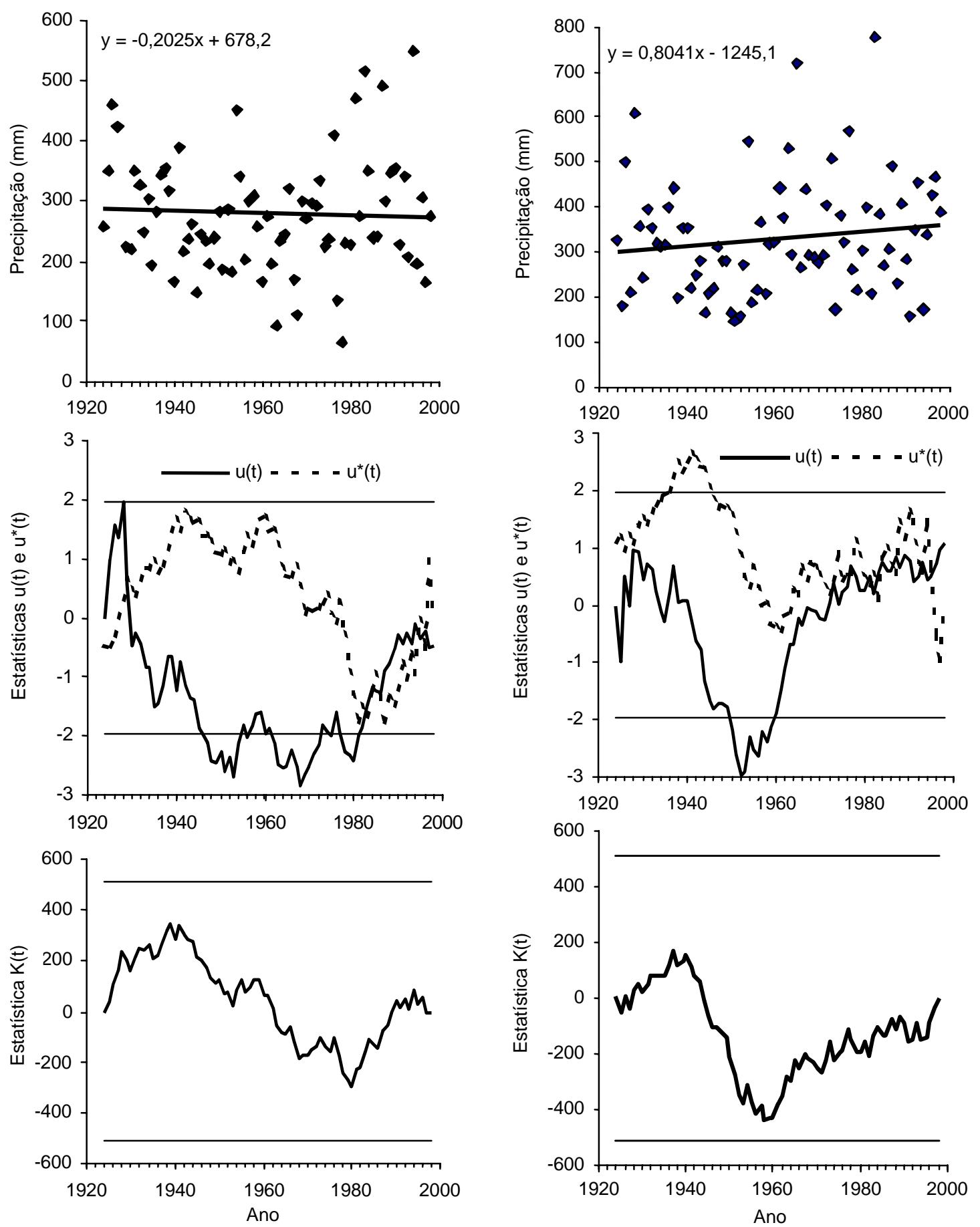

Figura 3. Precipitação total no segundo trimestre (regressão linear), estatísticas $\mathrm{u}(\mathrm{t})$ e $\mathrm{u}^{*}(\mathrm{t})$ do teste de MannKendall e estatística $\mathrm{K}(\mathrm{t})$ do teste de Pettitt. As linhas horizontais representam o intervalo de confiança de $95 \%$.

Figura 4. Precipitação total no terceiro trimestre (regressão linear), estatísticas $u(t)$ e $u^{*}(t)$ do teste de MannKendall e estatística $\mathrm{K}(\mathrm{t})$ do teste de Pettitt. As linhas horizontais representam o intervalo de confiança de $95 \%$. 

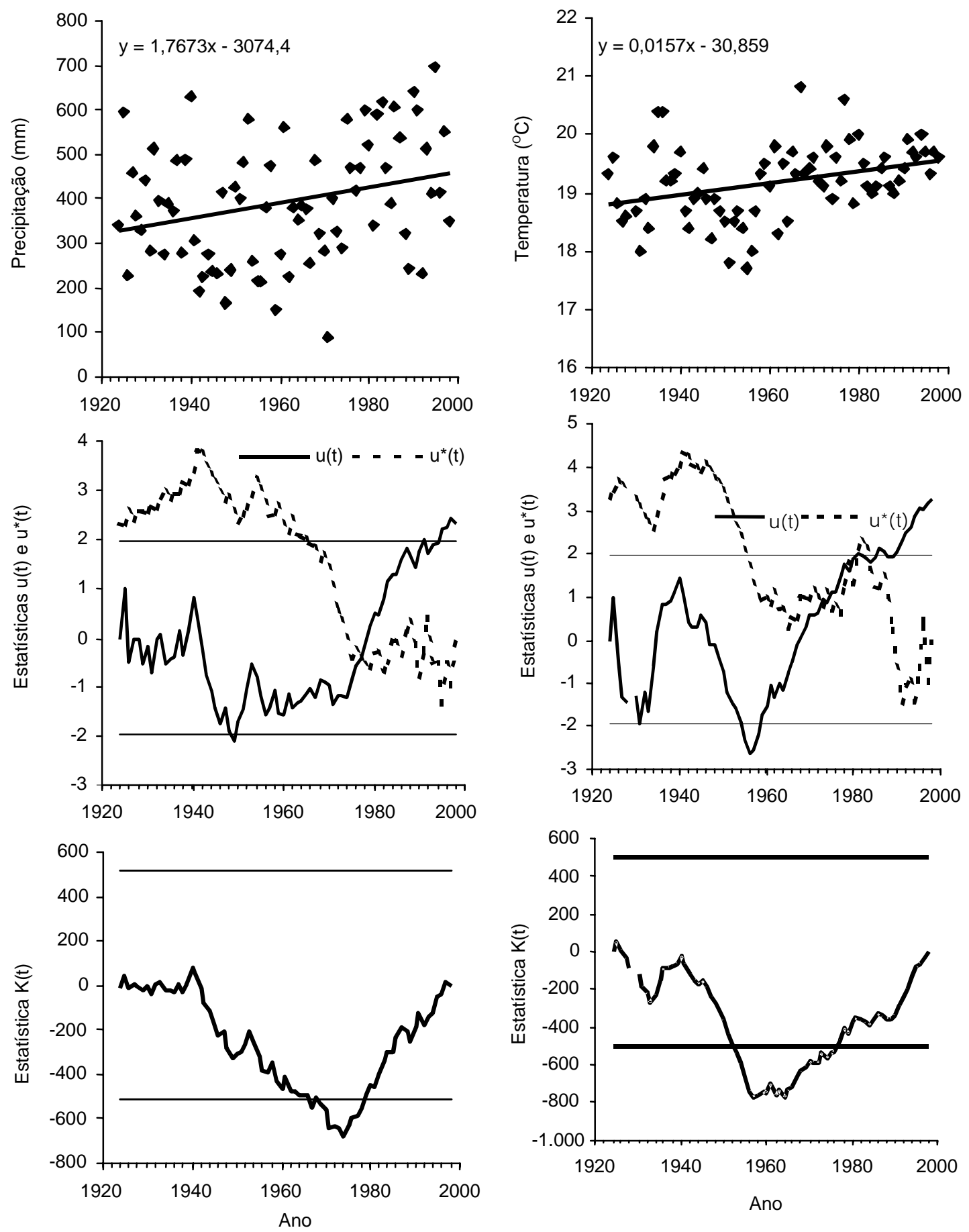

Figura 5. Precipitação total no quarto trimestre (regressão linear), estatísticas $u(t)$ e $u^{*}(t)$ do teste de MannKendall e estatística K(t) do teste de Pettitt. As linhas horizontais representam o intervalo de confiança de $95 \%$.

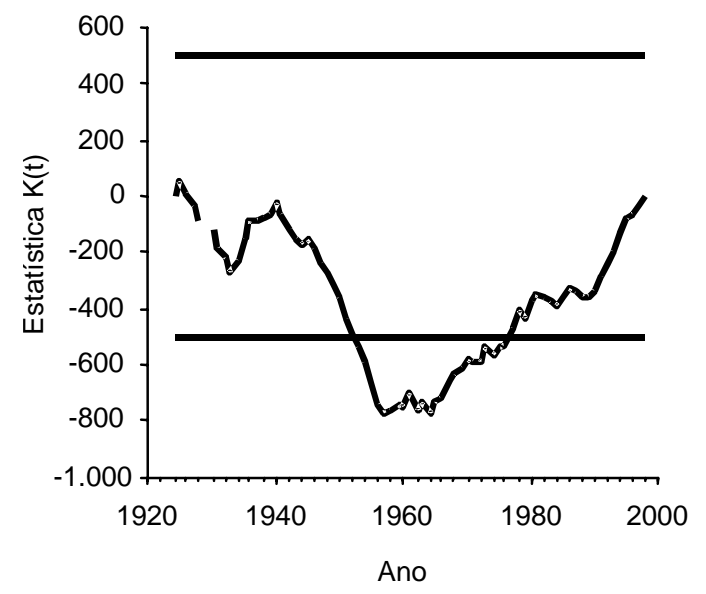

Figura 6. Temperatura média anual (regressão linear), estatísticas $u(t)$ e $u^{*}(t)$ do teste de Mann-Kendall e estatística $\mathrm{K}(\mathrm{t})$ do teste de Pettitt. As linhas horizontais representam o intervalo de confiança de $95 \%$. 

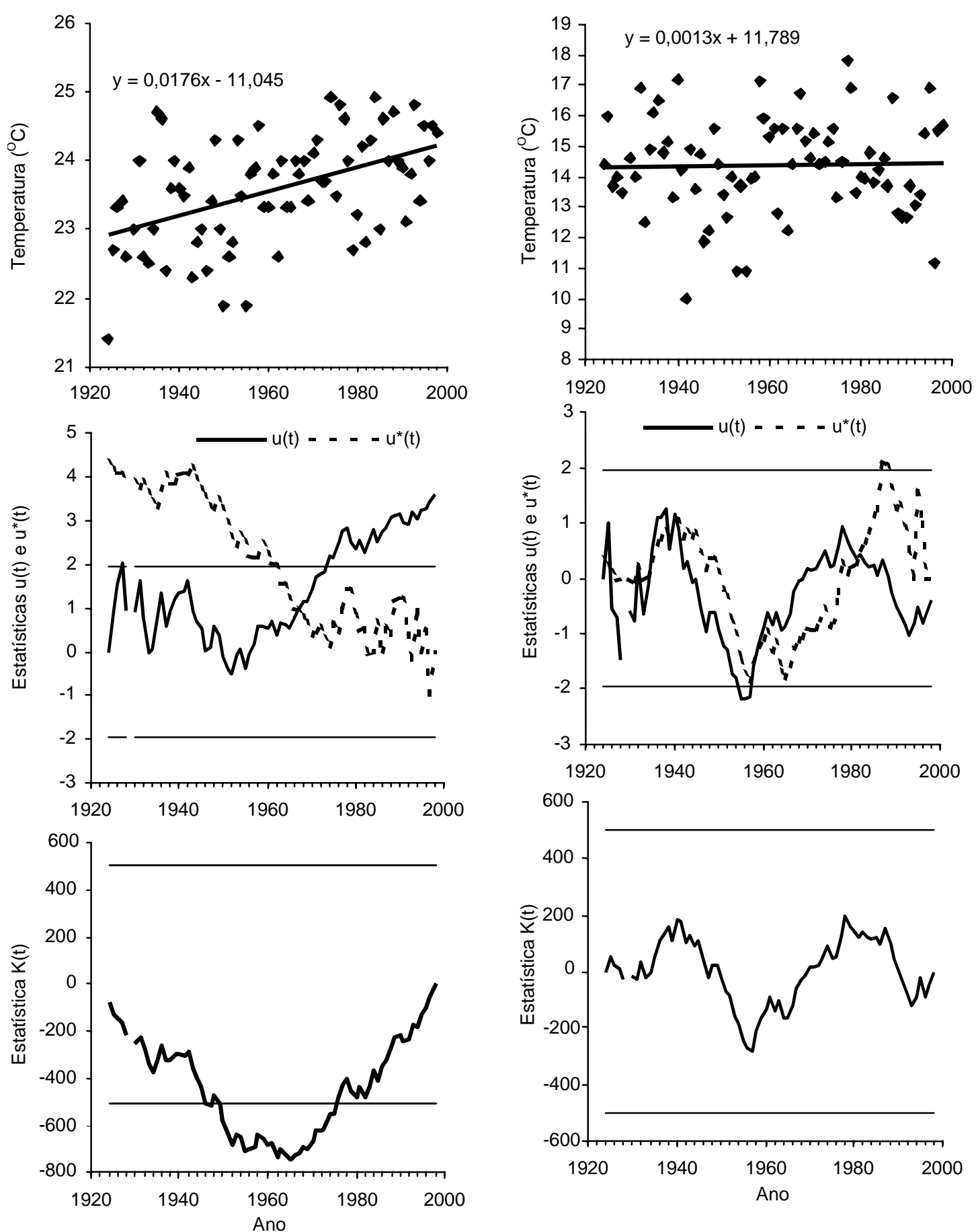

Figura 7. Temperatura média do mês de janeiro (regressão linear), estatísticas $\mathrm{u}(\mathrm{t})$ e $\mathrm{u}^{*}(\mathrm{t})$ do teste de Mann-Kendall e estatística $\mathrm{K}(\mathrm{t})$ do teste de Pettitt. As linhas horizontais representam o intervalo de confiança de $95 \%$.

Figura 8. Temperatura média do mês de julho (regressão linear), estatísticas $u(t)$ e $u^{*}(t)$ do teste de Mann-Kendall e estatística $\mathrm{K}(\mathrm{t})$ do teste de Pettitt. As linhas horizontais representam o intervalo de confiança de $95 \%$. 
limites de confiança e o início da mesma pode ser localizado pela interseção das curvas $u(t)$ e $u^{*}(t)$, se esta ocorre dentro dos valores críticos do intervalo de confiança. O teste de Pettitt indica o ponto de mudança brusca do valor máximo de $\mathrm{K}(\mathrm{t})$, quando este for maior que o limite crítico estabelecido.

Observa-se que a precipitação total anual apresentou uma oscilação com valores baixos entre os anos de 1942 a 1970. O teste de Pettitt indica que no ano de 1971 houve uma mudança com a tendência crescente da precipitação total anual, e pelo teste de Mann-Kendall observa-se que esta tendência tornou-se significativa no ano de 1998 (Figura 1). Estes dados estão coerentes com os estudos de Berlato et al. (1995), que encontraram, na média do Estado do Rio Grande do Sul, uma redução de 5,2 mm/ano na série pluviométrica de 1913 a 1951, compensada por um aumento de 5,6 mm/ano no período de 1952 a 1990. Esses resultados indicam uma tendência de flutuação da precipitação pluvial em curtos períodos.

O teste de Pettitt apresenta concordância com a análise de regressão, não identificando mudança brusca significativa nos dados de precipitação dos três primeiros trimestres. Já o teste de Mann-Kendall aponta períodos com tendência decrescente da precipitação, atingindo os limites de significância nos anos de 1943, 1945 e 1950. Na segunda metade do século, nenhuma tendência significativa na precipitação foi detectada (Figuras 2, 3 e 4).

O teste de Mann-Kendall indica que há tendência crescente da precipitação no quarto trimestre, tornando-se significativa no ano de 1991. Da interseção das linhas $\mathrm{u}(\mathrm{t})$ e $\mathrm{u}^{*}(\mathrm{t})$, observa-se que o início da mudança ocorreu no ano de 1976. Já o teste de Pettitt aponta para a mudança brusca significativa ocorrendo no ano de 1974 (Figura 5). A tendência crescente da precipitação pode ser influenciada pelos valores extremos registrados na década de 90, período em que ocorreram vários episódios de El Niño. Fontana \& Berlato (1997) mostraram que nos anos de ocorrência de El Niño são registrados no Rio Grande do Sul valores de precipitação pluvial acima da média no período de outubro a dezembro. Essa hipótese reforça a idéia de que a mudança na precipitação seja apenas uma oscilação de causa natural, não neces- sariamente associada a uma mudança definitiva nos padrões pluviométricos. Ayoade (1996) ressalta que flutuações no clima em períodos de 30 a 35 anos não podem ser consideradas como mudanças climáticas.

A temperatura média anual também apresenta tendência crescente, tornando-se significativa no ano de 1981, sendo que o início da tendência ocorre no ano de 1973 (Figura 6). A temperatura média apresentou valores crescentes no mês de janeiro, tornando-se significativa no ano de 1972, e o início da mudança ocorreu no ano de 1968 (Figura 7). Pelo teste de Pettitt, a mudança na temperatura média anual e temperatura média do mês de janeiro ocorreram no ano de 1965. Não foi identificada tendência significativa na temperatura média do mês de julho pelos teste de Pettitt e Mann-Kendall (Figura 8).

\section{Conclusões}

1. A análise de regressão, o teste de Pettitt e o teste de Mann-Kendall apresentam concordância entre seus resultados e podem ser utilizados na identificação de tendências de séries temporais.

2. O teste de Run não é adequado na identificação de tendências e mudanças climáticas.

3. A precipitação pluvial total anual apresenta tendência crescente dentro do período estudado; porém, quando analisada por trimestre, somente a precipitação pluvial no quarto trimestre apresenta tendência crescente.

4. A temperatura média anual e a temperatura média de janeiro apresentam tendência crescente, e a temperatura média de julho não apresenta tendência.

\section{Referências}

AYOADE, J. O. Introdução à climatologia para os trópicos. Rio de Janeiro : Bertrand Brasil, 1996. 332 p.

BERLATO, M. A.; FONTANA, D. C.; BONO, L. Tendência temporal da precipitação pluvial anual no Estado do Rio Grande do Sul. Revista Brasileira de Agrometeorologia, Santa Maria, v. 3, p. 111-113, 1995.

BRUCE, J. P. The atmosphere of the living planet earth. Genève : World Meteorological Organization, 1990. 42 p. (WMO, 735). 
DAI, A.; FUNG, I.; GENIO, A. D. del. Surface observed global land precipitation variations during 1900-1988. Journal of Climate, Boston, v. 10, p. 2946-2962, 1997.

FONTANA, D. C.; BERLATO, M. A. Influência do El Niño: oscilação sul sobre a precipitação pluvial do Estado do Rio Grande do Sul. Revista Brasileira de Agrometeorologia, Santa Maria, v. 5, n. 1, p. 127-132, 1997.

GOOSSENS, C.; BERGER, A. Annual and seasonal climatic variations over the northern hemisphere and Europe during the last century. Annales Geophysicae, Berlin, v. 4, n. B4, p. 385-400, 1986.

HANSEN, J.; LEBEDEFF, S. Global trends of measured surface air temperature. Journal of Geophysical Research, Washington, v. 92, n. 11, p. 13345-13372, 1987.

HOUGHTON, J. T.; CALLANDER, B. A.; VARNEY, S. K. (Ed.). Climate change 1992: the supplementary report to the IPCC scientific assessment. Cambridge (Inglaterra) : Cambridge University Press, 1992. 200 p.

HOUGHTON, J. T.; JENKINS, G J.; EPHRAUMS, J. J.(Ed.) Climate change: the IPCC scientific assessment. Cambridge (Inglaterra) : Cambridge University Press, 1990. 364 p.

HOUGHTON, J. T.; MEIRA FILHO, L. C.; CALLANDER, B. A.; HARRIS, N.; KATTERNBERG, A.; MASKELL, K. (Ed.). Climate change 1995: the science of climate change: contribution of working group I to the second assessment report of the Intergovernmental Panel on Climate Change. Cambridge (Inglaterra) : Cambridge University Press, 1996. 584 p.

JONES, P. D.; RAPER, S. C. B.; WIGEY, T. M. L. Southern hemisphere surface air temperature variations 1851-1984. Journal of Climate and Applied Meteorology, Boston, v. 25 , n. 9 , p. $1213-1230,1986$.

KARL, T. R.; DIAZ, H.; KUKLA, G Urbanization: its detection and effect in the United States climate record. Journal of Climate, Boston, v. 1, n. 11, p. 1099-1123, 1988.

KARL, T. R.; KNIGHT, R. W.; EASTERLING, D. R.; QUAYLE, R. G. Indices of climate change for the United States. American Meteorological Society Bulletin, Boston, v. 77, n. 2, p. 279-292, Feb. 1996.

MELLO, M. H. A.; ALFONSI, R. R. A variação térmica secular em Campinas, SP: um exemplo de problemas inerentes ao estudo de séries temporais em climatologia. In: CON-
GRESSO BRASILEIRO DE AGROMETEOROLOGIA, 7., 1991, Viçosa. Anais... Viçosa : Sociedade Brasileira de Agrometeorologia/UFV, 1993. p. 244-246.

MORAES, J. M.; PELLEGRINO, G.; BALLESTER, M. V.; MARTINELLI, L. A.; VICTORIA, R. L. Estudo preliminar da evolução temporal dos componentes do ciclo hidrológico da bacia do Rio Piracicaba. In: SIMPÓSIO BRASILEIRO DE RECURSOS HÍDRICOS, 11.; SIMPÓSIO DE HIDRÁULICA E RECURSOS HÍDRICOS DOS PAÍSES DE LÍNGUA OFICIAL PORTUGUESA, 2., 1995, Recife. Anais... Recife : Associação Brasileira de Recursos Hídricos, 1995. p. 27-32.

MOTA, F. S.; SIGNORINI, E.; ALVES, E. G. P.; AGENDES, M. O. O. Tendência temporal da temperatura no Rio Grande do Sul. Revista Brasileira de Agrometeorologia, Santa Maria, v. 1, n. 1, p. 101-103, 1993.

NATIONAL RESEARCH COUNCIL (Washington, Estados Unidos). Policy implications of greenhouse warming: mitigation, adaptation, and the science base. Washington : National Academy Press, 1992. 918 p.

PETTITT, A. N. A non-parametric approach to the changepoint problem. Applied Statistics, London, v. 28, n. 2, p. 126-135, 1979.

PRUDÊNCIO, R. S.; GEREMIAS, R.; VEADO, R. W. V. Análise do ritmo pluviométrico do litoral catarinense. In: CONGRESSO BRASILEIRO DE AGROMETEOROLOGIA, 11., 1999, Florianópolis. Anais... Florianópolis : Sociedade Brasileira de Agrometeorologia, 1999. p. 1055-1063.

SENTElhas, P. C.; CAMARGO, A. P.; CAMARGO, M. B. P.; ALFONSI, R. R. Um século de desmatamento: efeitos no regime térmico, pluvial e no balanço hídrico em Campinas, SP. Revista Brasileira de Agrometeorologia, Santa Maria, v. 2, p. 99-103, 1994.

SNEYERS, R. Sur l'analyse statistique des séries d'observations. Genève : Organisation Météorologique Mondial, 1975. 192 p. (OMM Note Technique, 143).

THOM, H. C. S. Some methods of climatological analysis. Genève: World Meteorological Organization, 1966. 54 p. (WMO Technical Note, 81).

YEVJEVICH, V. Probability and statistics in hydrology. Fort Collins : Water Resources Publication, 1972. 276 p. 